\title{
Genome-wide comparative analysis of DNA methylation between soybean cytoplasmic male-sterile line NJCMS5A and its maintainer NJCMS5B
}

Yanwei Li, Xianlong Ding, Xuan Wang, Tingting He, Hao Zhang, Longshu Yang, Tanliu Wang, Linfeng Chen, Junyi Gai and Shouping Yang*

\begin{abstract}
Background: DNA methylation is an important epigenetic modification. It can regulate the expression of many key genes without changing the primary structure of the genomic DNA, and plays a vital role in the growth and development of the organism. The genome-wide DNA methylation profile of the cytoplasmic male sterile (CMS) line in soybean has not been reported so far.

Results: In this study, genome-wide comparative analysis of DNA methylation between soybean CMS line NJCMS5A and its maintainer NJCMS5B was conducted by whole-genome bisulfite sequencing. The results showed 3527 differentially methylated regions (DMRs) and 485 differentially methylated genes (DMGs), including 353 high-credible methylated genes, 56 methylated genes coding unknown protein and 76 novel methylated genes with no known function were identified. Among them, 25 DMRs were further validated that the genome-wide DNA methylation data were reliable through bisulfite treatment, and 9 DMRs were confirmed the relationship between DNA methylation and gene expression by qRT-PCR. Finally, 8 key DMGs possibly associated with soybean CMS were identified.

Conclusions: Genome-wide DNA methylation profile of the soybean CMS line NJCMS5A and its maintainer NJCMS5B was obtained for the first time. Several specific DMGs which participated in pollen and flower development were further identified to be probably associated with soybean CMS. This study will contribute to further understanding of the molecular mechanism behind soybean CMS.
\end{abstract}

Keywords: Soybean (Glycine max (L.) Merr.), Cytoplasmic male sterility, DNA methylation, Differentially methylated gene, Gene expression

\section{Background}

Cytoplasmic male sterility (CMS) is a common maternally inherited phenomenon that prevents the production of functional pollen [1]. In most cases of CMS, male-fertility can be recovered by specific gene named as restorer-of-fertility (RF) in the nuclear genome [2,3]. At present, CMS has been present in more than 200

\footnotetext{
* Correspondence: spyung@126.com

Soybean Research Institute, National Center for Soybean Improvement, Key Laboratory of Biology and Genetic Improvement of Soybean (General, Ministry of Agriculture), State Key Laboratory of Crop Genetics and Germplasm Enhancement, Jiangsu Collaborative Innovation Center for Modern Crop Production, Nanjing Agricultural University, Nanjing 210095,
} China

species of plants [4] and was widely used in crops hybrid [6], micreen reported in transcriptomics [5], proteomics the epigenetic regulation of the CMS remains poorly derstood.

DNA methylation, a conserved epigenetic silencing mechanism in most eukaryote, could regulate the expression of many key genes, for example histone methylation lation repressing the LDMAR gene expression [10], and FLC chromatin with methylated modification delaying flowering [11]. In plant and animal, DNA methylation 
occurs predominately in CG enriched region of the genome, especially at CG island. However, cytosine methylation has also been observed in the CHG and $\mathrm{CHH}$ contexts with a low level in plant genome [12]. According to statistics, DNA methylation level varies from $4.6 \%$ to $30 \%$ in plant, which is relatively higher than that in animal [13]. No matter animal, plant or fungi, active genes are generally unmethylated, while transposable elements (TEs) are heavily methylated. So it was proposed that DNA methylation is roughly positively correlated with TE abundance, but negatively related to gene expression [14]. Within promoter region, DNA methylation is supposed to impact the transcriptional level of genes by silencing TE; whereas, within the gene body, DNA methylation may be associated with highly-expressed genes [15]. In addition, the establishment of DNA methylation mainly depends on DNA methyltransferases (DNMT), and DNA methylation regulation also relies on DNMT. To date, genes that encode DNMT have been isolated from a variety of plants, including rice, tobacco, corn and soybean [16-20].

Whole-genome sequencing and methylation profile has been widely reported in many plants, such as Arabidopsis [15], rice [21], soybean [14] and cotton [22]. Chen et al. [23] studied differentially methylation levels between the rice male sterile line PA64s and its fertile plant, and found the methylation level was higher in the sterile line than that of the fertile line. In addition, they also identified a differentially methylated gene (DMG) that was probably related to male sterility [24]. Liu et al. [25] proposed that the DNA methylation levels were higher in the corn CMS line than that in the maintainer line, and inferred that the changes of DNA methylation level in maize tassel may be associated with CMS. Although DNA methylome has also been used to analyze the distribution and average level of methylation in soybean root, stem, leaf and cotyledon [14], how DNA methylation may regulate the soybean CMS has no related report so far.

The soybean CMS line NJCMS5A was developed through consecutive backcross procedures with NJCMS1A [26-28] as the donor parent and Wandou 28 (designated as NJCMS5B afterwards) as recurrent parent in our laboratory. In this study, genome-wide comparative analysis of DNA methylation between soybean CMS line NJCMS5A and its maintainer NJCMS5B was conducted by wholegenome bisulfite sequencing. This is the first time to exploit epigenetic variation, and how gene expression is regulated in the whole genome of the soybean CMS.

\section{Results}

\section{Analysis of genome-wide DNA methylation data of} NJCMS5A and NJCMS5B

To study the genome-wide DNA methylation pattern of soybean, we collected flower buds from the soybean CMS line NJCMS5A and its maintainer NJCMS5B for constructing genomic DNA libraries. In total, 383,901,574 (NJCMS5A) and 398,207,546 (NJCMS5B) raw reads were generated from the two DNA library samples by whole-genome bisulfite sequencing (Table 1). After removal of related adapters, low-quality reads and containing Ns, $147,400,718$ in NJCMS5A and 150,714,851 in NJCMS5B clean reads were collected (Table 1), of which $65.08 \%$ (NJCMS5A) and 62.10\% (NJCMS5B) were uniquely mapped to the reference soybean genome of Williams82 (https://phytozome.jgi.doe.gov/pz/ portal.html\#! info?alias=Org_Gmax) (Table 1). Over $88 \%$ cytosines in soybean genome were covered by at least 5-fold coverage (Additional file 1). Meanwhile, more than $99 \%$ cytosines were converted, which indicates a high conversion rate in this study (Table 1).

The difference of DNA methylation level in the genome-wide between NJCMS5A and NJCMS5B was not significant. We detected $14.60 \%$ and $14.26 \%$ of methylated cytosines in NJCMS5A and NJCMS5B (Fig. 1a). Meanwhile, the average level of methylated cytosines in each context was also calculated for them. There were $48.58 \%$ of mCG (mCG/CG), 39.10\% of mCHG (mCHG/ $\mathrm{CHG})$ and $6.74 \%$ of $\mathrm{mCHH}(\mathrm{mCHH} / \mathrm{CHH})$ in NJCMS5A. Correspondingly, 47.37\%, 37.27\% and 6.73\% of cytosines were methylated in CG, CHG and $\mathrm{CHH}$ contexts of NJCMS5B, respectively (Fig. 1a). Clearly, the average levels of mCG and mCHG were much higher than that of $\mathrm{mCHH}$ (Fig. 1a). And this distribution trend in three contexts in flower bud is generally similar to the trend reported previously in soybean root, steam, leaf and cotyledon [14]. Surprisingly, though the percent of mCHG was much lower than that of $\mathrm{mCG}$ and $\mathrm{mCHG}$, the number of methylated cytosine sites in the three contexts was very similar. In NJCMS5A, we detected 48,495,434 mC sites, $15,497,738$ mCG cites (32.0\% of all $\mathrm{mC}), \quad 15,425,507 \mathrm{mCHG}$ sites $(31.8 \%$ of all $\mathrm{mC})$, $17,572,189 \mathrm{mCHH}$ sites $(36.2 \%$ of all $\mathrm{mC}$ ), respectively; similarly, there were $31.9 \% \mathrm{mCG}, 31.1 \% \mathrm{mCHG}$, and $37.0 \% \mathrm{mCHH}$ in NJCMS5B (Fig. 1b). These results are consistent with the previous report about the DNA methylome of soybean LD00-2817P (“LD”) germplasm

Table 1 Summary of genome-wide methylation sequencing data

\begin{tabular}{lllllll}
\hline Sample & Raw reads & $\begin{array}{l}\text { Clean } \\
\text { reads }\end{array}$ & $\begin{array}{l}\text { Total unique } \\
\text { mapped reads }\end{array}$ & $\begin{array}{l}\text { Percentage of mapped } \\
\text { reads in total reads }\end{array}$ & $\begin{array}{l}\text { The coverage of } \\
5 \times \text { reads in total reads }\end{array}$ & $\begin{array}{l}\text { Bisulfite sequencing } \\
\text { conversion rate }\end{array}$ \\
\hline NJCMS5A & $383,901,574$ & $147,400,718$ & $95,930,507$ & $65.08 \%$ & $88.75 \%$ & $99.75 \%$ \\
NJCMS5B & $398,207,546$ & $150,714,851$ & $93,590,676$ & $62.10 \%$ & $88.48 \%$ & $99.39 \%$ \\
\hline
\end{tabular}




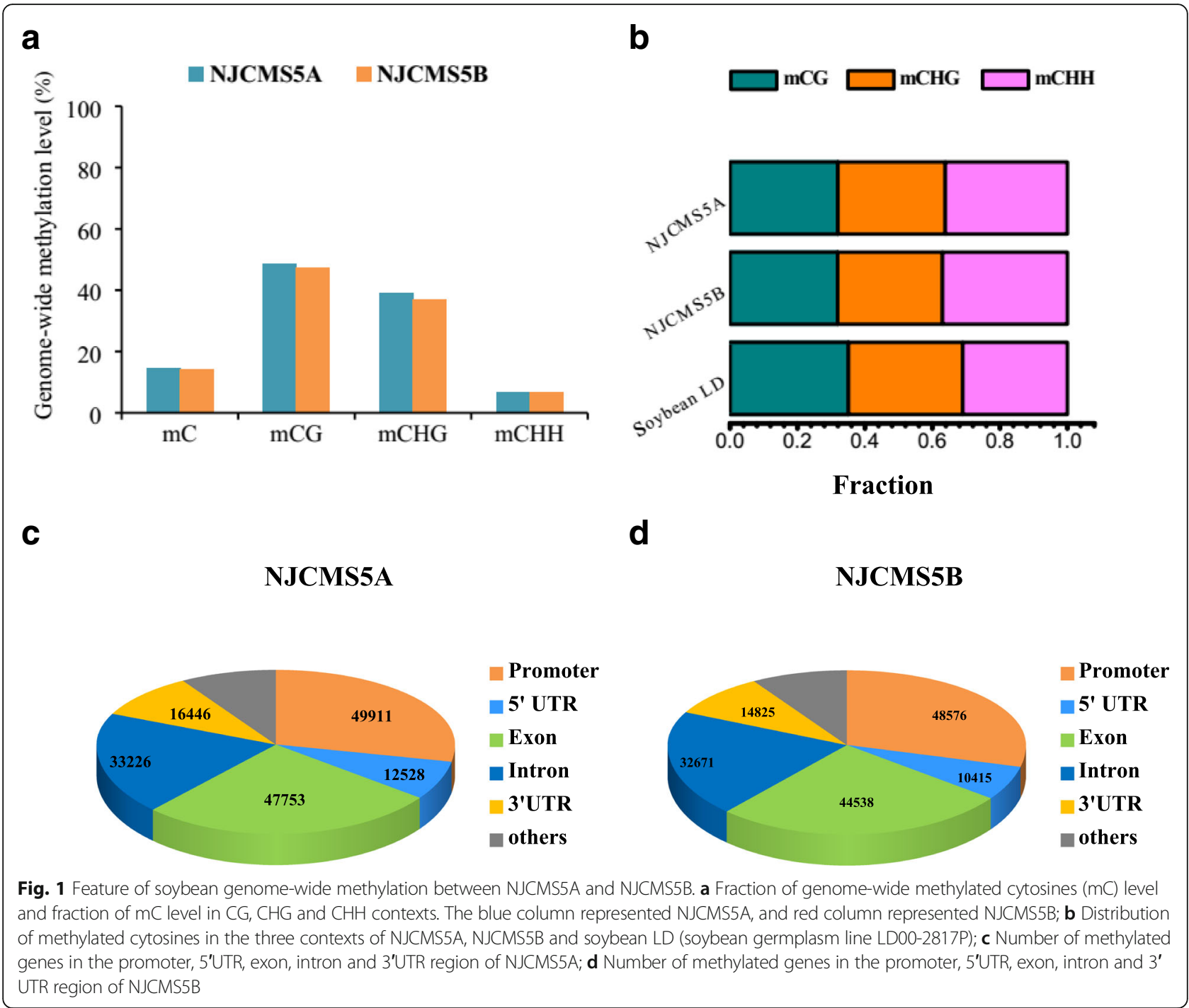

(Fig. 1b) [29]. So, we inferred there was a sizable proportion in of non-CG (CHG and $\mathrm{CHH})$ in the plant cell.

\section{Profile of DNA methylation in gene and transposable element (TE) region}

The density distribution of methylated cytosines was tested to detect DNA methylation region. Firstly, from the chromosome level, DNA methylation was enriched mostly in the centromeric region, but with a little methylation was present at both ends of the chromosome (Additional file 2). Secondly, to better understand the relationship of DNA methylation and gene expression, we divided the genome into some functional regions, namely promoter region defined as the $2 \mathrm{~kb}$ region upstream of a transcription start site (TSS) and gene body consisting of 5'UTR, exon, intron and 3' UTR. For the promoter region, the DNA methylation level rapidly increased as departing from the TSSs sites in all contexts (Fig. 2). And it showed a low level in the gene body (Fig. 2). Besides this feature, when compared with $\mathrm{CHH}$ methylation level, $\mathrm{CG}$ and $\mathrm{CHG}$ methylation levels were significantly higher in promoter or gene body region (Fig. 2).

To further assess the correlations among DNA methylation, TE and gene expression, we collected genome sequences (from our laboratory) and TE sequences from SoyTEdb (http://www.soybase.org/soytedb/) [30, 31] (Fig. 3). On the whole, the pattern of DNA methylation in NJCMS5A was very similar to that in NJCMS5B (Figs. 2 and 3). The level of DNA methylation of the whole soybean genome was just about 14\%. Except for C-G nucleotide pairs, most methylation occurred in CHG site (Figs. 1a and 3). In the $\mathrm{CG}, \mathrm{CHG}$ and $\mathrm{CHH}$ contexts, DNA methylation was most abundant in TE-rich and gene-poor expression region in this study (Fig. 3). So DNA methylation 

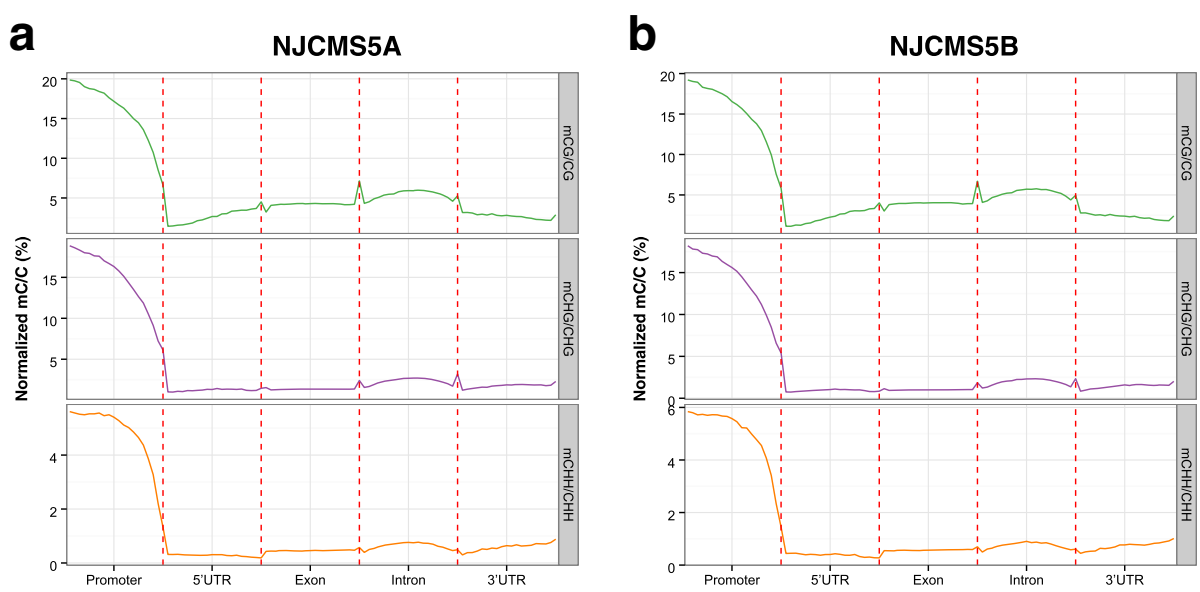

Fig. 2 DNA methylation pattern in gene functional region under $m C, m C G, m C H G$ and $m C H H$ contexts. a Average density of DNA methylation in the promoter, 5'UTR, exon, intron and $3^{\prime} U T R$ region under three contexts of NJCMS5A; $\mathbf{b}$ Average density of DNA methylation in the promoter, 5' UTR, exon, intron and $3^{\prime} U T R$ region under three contexts of NJCMS5B. Each functional region was equally divided into 20 bins, and the mean of methylated cytosine density in each bin was defined as methylation density

may be positively related with TE density and negatively related to the expression level of genes in the soybean genome, which agrees with an idea proposed previously [29]. However, more empirical evidence is required to confirm that this hypothesis could be applied to any $\mathrm{mC}$ site detected in this study.

\section{Effect of DNA methylation on gene expression}

Considered that the higher methylation level may reduce gene expression in the promoter region, we performed a further analysis of gene expression in different functional region using RNA-Seq data. Genes were divided into five groups, according to their expression level (Additional

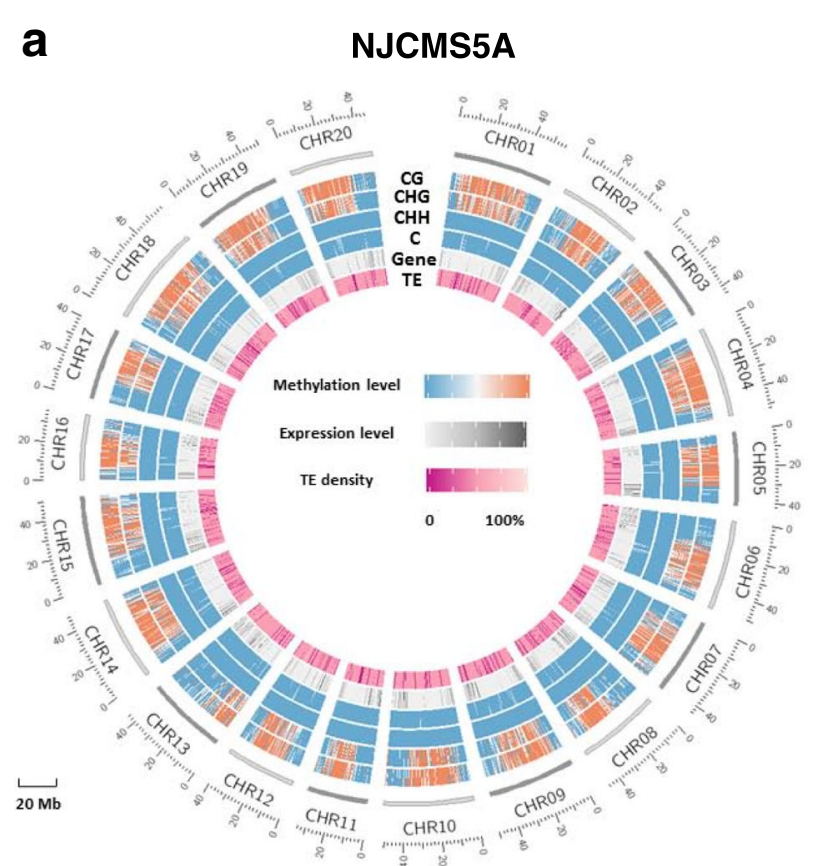

b

NJCMS5B

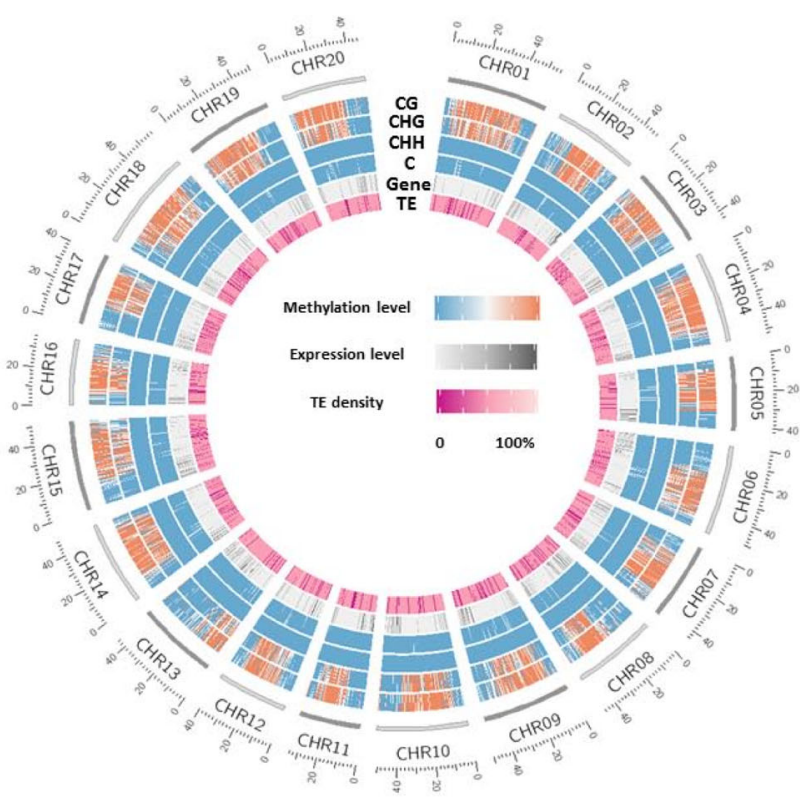

Fig. 3 Circle plots of gene expression level, transposable element (TE) density and methylation level in the mC, mCG, mCHG and mCHH contexts of soybean (a) NJCMS5A and (b) NJCMS5B. TE indicated "TE density"; Gene indicated "gene expression level"; C, CG, CHH and CHG indicated "methylation level in $\mathrm{mC}, \mathrm{mCG}, \mathrm{mCHG}$ and $\mathrm{mCHH}$, respectively". Gene expression level was calculated by fragments per kilobase of exon model per million mapped fragments (RPKM). Data is plotted for $20 \mathrm{Mb}$ windows in all soybean chromosomes (CHRO1-CHR20). Gene expression level (from light colour to dark colour) indicates the level from 0 to 100\%, and TE density (from dark colour to light colour) indicates the density from 0 to $100 \%$ 
file 3): 0-1 (silent gene), 1-3 (low expressed gene), 3-15 (moderately expressed gene), 15-60 (highly expressed gene) and $>60$ (gene with highest expression). The results clearly showed the negative correlation between DNA methylation and gene expression level in the promoter (Fig. 1c, d and Additional file 4). Due to large amounts of methylated genes (49,911 of NJCMS5A, 48,576 of NJCMS5B) occurred in promoter region, gene expression level in promoter was obviously lower than that in gene body (Fig. 1c, $d$ and Additional file 4). However, in the gene body, the expression levels of high and middle genes were much high, which indicated no negative or positive correlation between gene expression and DNA methylation (Fig. 1c, d and Additional file 4).

\section{Identification of differentially methylated gene (DMG) between NJCMS5A and NJCMS5B}

Differentially methylated region (DMR) is a significant sign of epigenetic variation, which may participate in regulating the DMGs to influence biological processes. By a genome-wide comparison of DNA methylation sequences between NJCMS5A and NJCMS5B, 3527 DMRs (Additional file 5) were identified by swDMR software (https://sourceforge.net/projects/swdmr/) with "FDR s 0.05 and coverage changes $\geq 5$ " in this study. Then, DMGs were defined as genes overlapping with significant DMRs with at least $1 \mathrm{bp}$ in the promoter and/or gene body. From 739 DMRs (Additional file 6) with genomic feature, we obtained 485 non-repeat DMGs including 281 hypo-methylated genes (57.9\%) and 88 hypermethylated genes $(18.2 \%)$ in the NJCMS5A relative to NJCMS5B. In addition, 116 methylation-unstable genes (23.9\%) that overlapped with different gene functional region (promoter, 5'UTR, exon, intron or 3'UTR) showed both hyper-methylation and hypo-methylation (Fig. 4a). In the gene functional region, there were more promoter-related DMGs (59.2\%) than the exon-related

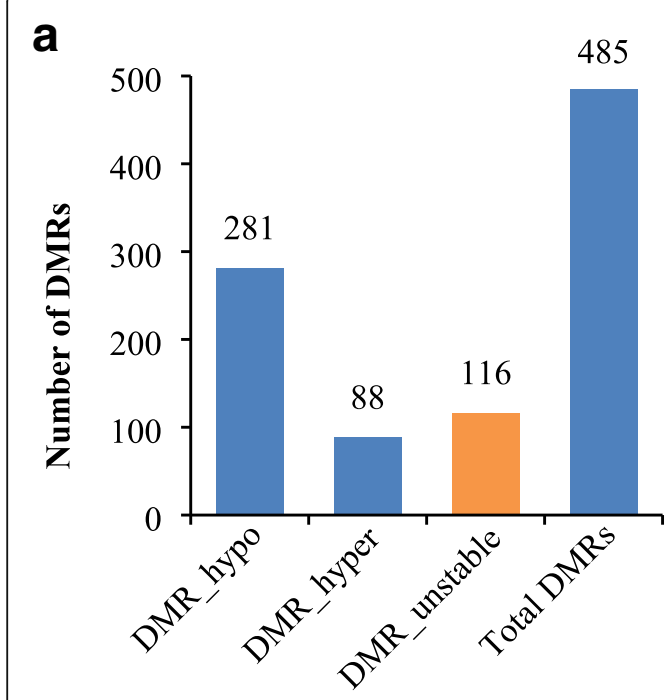

b

C

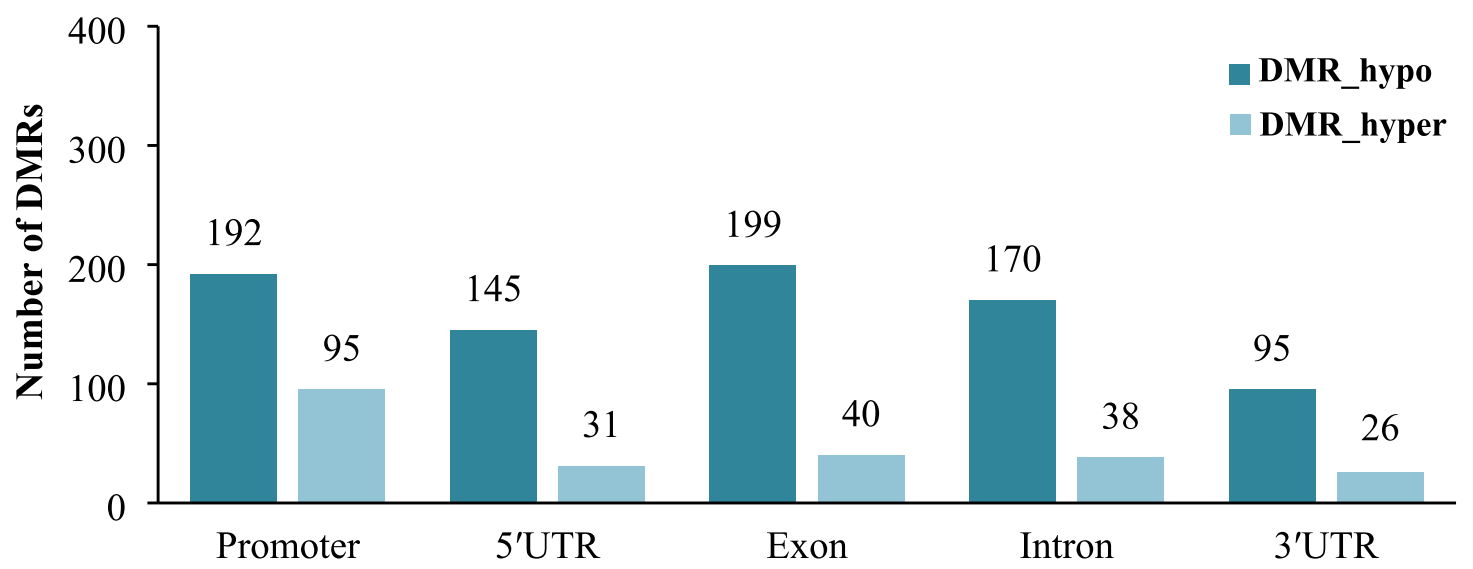

Fig. 4 Distribution and proportion of differentially methylated genes (DMGs). a Total DMGs divided into hypo-DMGs and hyper-DMGs and unstable-DMGs (genes overlapping with both hyper- and hypo- DMGs); b Number of DMGs in gene functional region (promoter, 5'UTR, exon, intron and $3^{\prime} U T R$ ); c Number of hypo-DMGs and hyper-DMGs in gene functional region (promoter, 5'UTR, exon, intron and $3^{\prime} U T R$ ) 
(49.3\%) or intron-related DMGs (42.9\%) (Table 2 and Fig. 4b). In addition, we found the number of hypomethylated DMGs was almost two to five times more than the number of hyper-methylated DMGs (Fig. 4c), which implied that DNA hypo-methylation often occurred as the epigenetic initial abnormality.

\section{Validation of the whole-genome bisulfite sequencing (WGBS) data by bisulfite treatment}

We randomly selected 25 DMRs (16 hypo-DMRs and 9 hyper-DMRs) in NJCMS5A to confirm the WGBS data by bisulfite treatment, including Glyma.04G206000 (OSBP), Glyma.19G144200 (bHLH DNA-binding), Glyma.16G081800 (lipase), Glyma.06G266900 (galacturonosyl transferase) and so on (Figs. 5 and 6). Many of DMGs showed the cytosines in the CG and CHG contexts were more frequently methylated than that in the $\mathrm{CHH}$ context (Fig. 5 and Additional file 7). The bisulfite sequencing results showed that 21 DMRs containing of 13 hypo-DMRs and 8 hyper-DMRs were consistent with the WGBS data, which indicated WGBS data were credible in the study (Additional files 7 and 8). In term of CG context, many DMGs were methylated highly, so we also made models of the methylated percentage at each site (Fig. 6), including Glyma.08G317600 (MYB domain protein), Glyma.10G248800 (methyltransferases), Glyma.U028100 (MYB domain protein) and Glyma.U013000 (AGAMOUS). Among them, the gene of Glyma.U013000 exhibited a higher methylation level in NJCMS5A compared NJCMS5B. But Glyma.08G317600, Glyma.10G248800 and Glyma.U028100 were identified to be hypo-methylation in NJCMS5A.

Gene ontology (GO) annotation and Kyoto encyclopedia of genes and genomes (KEGG) pathway enrichment analysis

To describe property of genes and their products, WEGO (http://wego.genomics.org.cn) was used to functionally categorize the DMGs (Fig. 7). In total of 334 DMGs, comprising of 270 hypo-methylated and 139 hyper-methylated DMGs were annotated to 31 functional categories, including, 14 biological processes (BP), 9 cellular components (CC) and 8 molecular functions (MF) (Fig. 7). In the term of BP, we found that the hyper-methylated DMGs were mainly involved in catabolic

Table 2 Distribution of differentially methylated genes (DMGs) in gene functional region

\begin{tabular}{llllll}
\hline Gene functional region & Promoter & 5' UTR $^{\prime}$ & Exon & Intron & 3' UTR \\
\hline DMG_hypo & 192 & 145 & 199 & 170 & 95 \\
DMG_hyper & 95 & 31 & 40 & 38 & 26 \\
Total DMG & 287 & 176 & 239 & 208 & 121 \\
DMG rate in genome (\%) & $59.2 \%$ & $36.3 \%$ & $49.3 \%$ & $42.9 \%$ & $24.9 \%$ \\
\hline
\end{tabular}

process (GO: 0044238, GO: 0044237, GO: 0043170), cellular localization (GO: 0051641), macromolecule of localization (GO: 0033036) and transport (GO: 0006810) (Fig. 7). In the term of CC, the hyper-methylated DMGs were mostly participated in nucleoplasm part (GO: 0005654, GO: 0044451), sperm part (GO: 0036126, GO: 0097223, GO: 0097228) and organism membrane (GO: 0033644, GO: 0044218, GO: 0044279). And in the term of MF, the hyper-methylated DMGs were mostly involved in the process of transcription regulation (GO:0060089) and transporter (GO:0005215, GO:0022857) (Fig. 7). In addition, those hyper-methylation genes of NJCMS5A were made for GO enrichment analysis by using the GOseq $\mathrm{R}$ package. The result showed many of them appeared to be enriched in macromolecule modification, cellular metabolism, transcription regulation, protein phosphorylation and so on. These corresponding genes were taken into account for significantly down-regulated genes and further were listed (Table 3), including 86 genes for "BP" (36), 15 genes for "CC" (17) and 95 genes for "MF" (41).

Among the DMGs, 51 DMGs (Additional file 9) were predicted to be enriched in 35 biochemical metabolic pathways in the KEGG database (http://www.genome.jp/kegg/), including plant hormone signal transduction (ko04075), glycolysis / gluconeogenesis (ko00010), regulation of actin cytoskeleton (ko04810), RNA degradation (ko03018), cysteine and methionine metabolism (ko00270) and oxidative phosphorylation (ko00190). Based on the GO and KEGG pathway analysis, 8 genes were determined to be potentially related to soybean CMS (Table 4). For example, anther wall tapetum development gene Glyma.U015500, ATPase activity genes Glyma.16G195100 and Glyma.06G248800, proteolysis gene Glyma.U045200, regulation of transcription genes Glyma.08G317600, Glyma.U028100 and Glyma.U040000 and mitochondrion structural gene Glyma.14G212600.

\section{Validation of target DMGs by quantitative real-time PCR (qRT-PCR)}

We also tried to conduct qRT-PCR to validate the relationship between DNA methylation and gene expression with 9 DMGs. As a result, 8 of 9 DMGs showed a negative correlation between DNA methylation and gene expression (Fig. 8). When the NJCMS5A methylation level of a DMG was significantly higher than that in NJCMS5B $(P<0.001)$ (Fig. 8b), the expression of the gene showed down-regulated in NJCMS5A, including Glyma.14G212600 (PPR protein), Glyma.U029400 (adenosine kinase) and Glyma.U040000 (AP2/B3 transcriptional factor protein). In addition, when the methylation level of a DMG in NJCMS5A was lower than that in NJCMS5B, the expression of the gene showed upregulated in NJCMS5A, such as Glyma.06G248800 (ABC transporter protein), Glyma.U045200 (cysteine proteinases protein), Glyma.06G266900 (galacturonosyl 

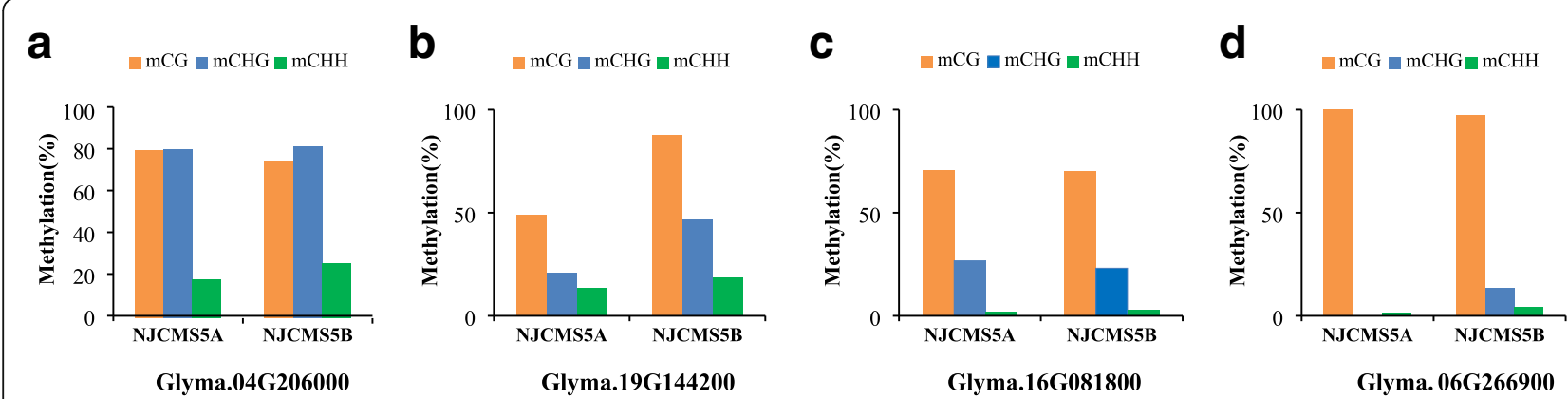

Fig. 5 DNA methylation patterns of four differentially methylated genes (DMGs) validated by bisulfite treatment. a Glyma.04G206000 (oxysterol binding protein); b Glyma.19G144200 (basic helix-loop-helix DNA-binding protein); c Glyma.16G081800 (lipase); d Glyma.06G266900 (galacturonosyl transferase). The red, blue and green columns in the histograms refer to the collective methylation levels (in percentage) in CG, $\mathrm{CHG}$ and $\mathrm{CHH}$ contexts, respectively

transferase), Glyma.08G305500 (fimbrin-like protein) and Glyma.16G195100 (mitochondrial mRNA modification). Only gene Glyma.U013000 (AGAMOUS) had a high DNA methylation level and showed a high expression in NJCMS5A, which may be related to its function of inhibiting the down-stream gene expression, and it would be verified through the further research.

\section{Discussion}

Although genome-wide DNA methylation map has been reported in many organism [15, 21, 22], DNA methylome of soybean CMS has few related studies. In this study, we apply DNA methylation sequencing on the soybean CMS line NJCMS5A and its maintainer NJCMS5B for the first time. And the methylation profile of flower bud was in line with the previously reported methylation profile of soybean root, steam, cotyledon and leaf [14]. The genomic DNA methylation data were obtained by wholegenome bisulfite sequencing from NJCMS5A and NJCMS5B (Table 1 and Fig. 1a), and it revealed that DNA methylation often occurred not only in CG context but also in non-CG ( $\mathrm{CHG}$ and $\mathrm{CHH}$ ) context throughout all chromosomes or genome functional region of soybean (Fig. 2), which is consistent with the DNA methylation level reported in other plants $[14,15,23,29]$.

DNA methylation is treated as an important epigenetic process that influences gene expression $[9,11]$. In this study, the global methylation patterns of the two soybean lines are in agreement with previous observations $[14,29]$. In addition, to further verify DNA methylation could silence gene expression in NJCMS5A, we select 9 DMRs to verify the negative correlation between DNA methylation and gene expression by qRT-PCR (Fig. 8). In addition, we identified 739 DMRs (Additional file 6) with genomic feature and 485 DMGs with 281 hypomethylated genes (57.9\%) and 88 hyper-methylated genes $(18.2 \%)$ in the NJCMS5A relative to NJCMS5B (Fig. 4a). According to the gene region annotation, gene function, GO and KEGG pathway analysis, as well as previously reported studies of male sterility in plant, 8 key DMGs (Table 4) that may be related to soybean CMS were identified. And they were further discussed as follows.

\section{DMGs involved in regulation of pollen development potentially related to CMS}

Numerous studies have shown that the pollen wall plays an important part in protecting pollen development [32]. The innermost layer of the anther wall (called tapetum) directly wrapped the microspore mother cell and microspore, and provided nutrition for microspore development by secreting large amounts of carbohydrate and lipid [33]. Therefore, tapetum played an important role in the process of pollen development, and the tapetum mutation may lead to pollen abortion [34-37]. The dysfunctional Tapertum1 gene (DYT1), encoding a basic helix-loophelix (bHLH) TF, participated in tapetum development and protection [38]. In Arabidopsis Dyt1 mutant, because of the thicker callose wall, the pollen mother cell was unable to produce spores, leading to pollen abortion. In addition, the transcriptome analysis of Arabidopsis wild-type and dyt1 mutant showed dyt1 gene was in upstream of at least 22 coding bHLH TF genes, participating in regulating many specific metabolic pathways [39]. In this study, a DMG Glyma.U015500 was found to encode a bHLH TF, which was a homolog of DYT1 in Arabidopsis. We supposed that Glyma.U015500 may participate in the formation of pollen wall and may have an impact on pollen development in soybean.

The pollen coat is the outermost layer of the anther and it is mainly composed of sterol esters to protect the pollen [40]. The ATP binding cassette (ABC) transporter 


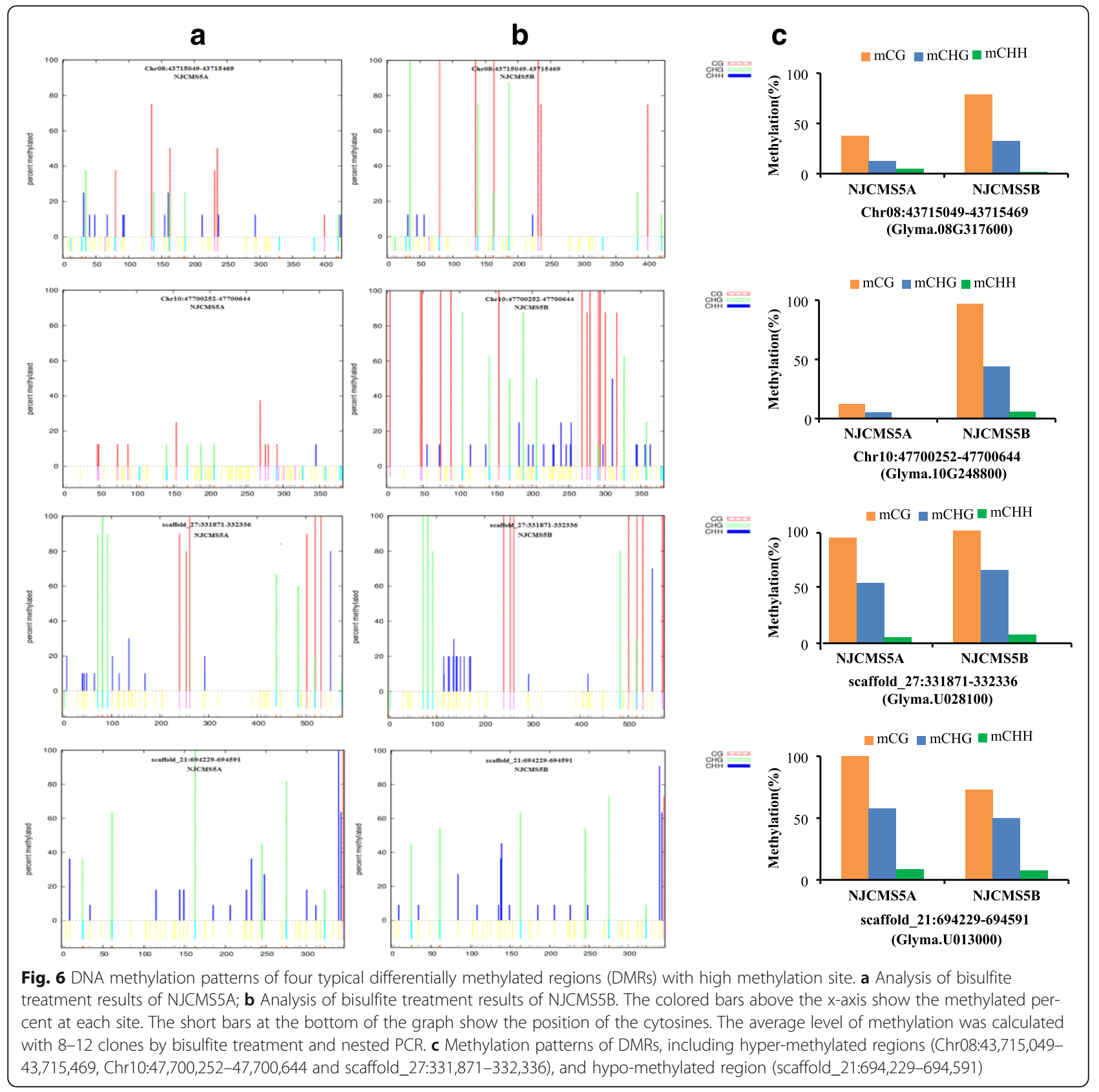

is in charge of transporting the coat materials for male gametophyte development [41]. In this study, a DMG Glyma.06G248800 was supposed to encode an ABC-2 type transporter family protein, which was a homolog of ABCG9 of Arabidopsis. ABCG9 mainly participates in formation of the pollen coat with a high expression level in tapetum. However, mutations of $a b c g 9-1$ and abcg31-1 produced a lot of distorted and shrunken pollen grains, without mature pollen released [42]. So we considered Glyma.06G248800 may contribute to the accumulation of sterol esters on the surface of the soybean pollen.
Together, abnormal methylation levels of Glyma.U015500 and Glyma.06G248800 in NJCMS5A may influence the development of the pollen wall, which may be a factor in the soybean CMS.

\section{DMGs involved in carbohydrate and energy metabolism potentially related to CMS}

In the early stage of development, pollen is surrounded by tapetum. And in the late stage, tapetum begins to degrade [43]. Tapetum programmed cell death (PCD) plays a vital role in developing pollen. Cysteine Protease participates in plant PCD as the most common hydrolytic 


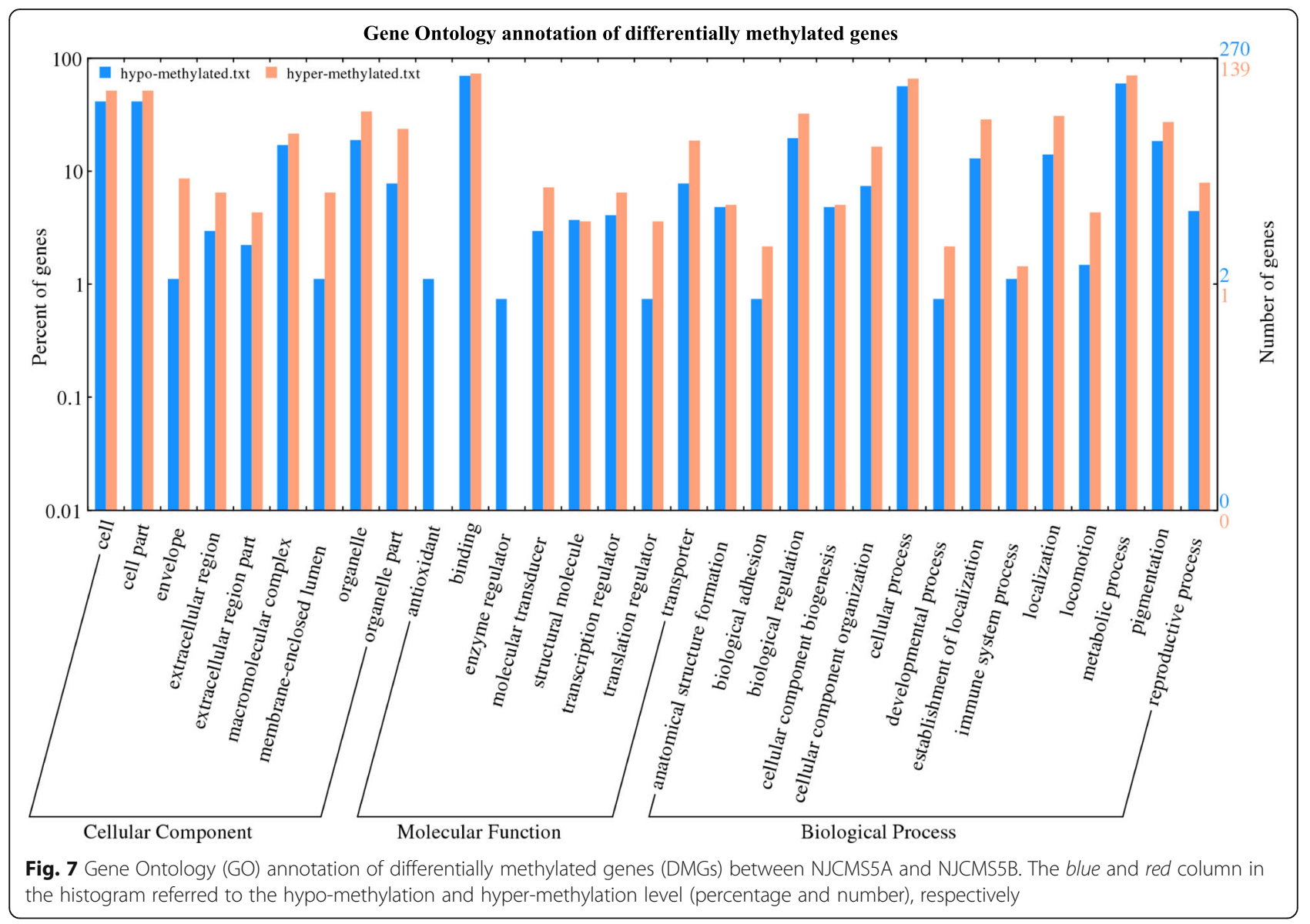

enzyme [44]. In this study, a DMG Glyma.U045200 was predicted to encode a homolog of Cysteine Protease CEP1 of Arabidopsis. CEP1 participates in tapetum PCD and regulates the expression of FLOWERING LOCUS T (FT) in Arabidopsis. Excessive expression of CEP1 led to tapetum PCD degeneration in advance and pollen abortion [45], so proper CEP1 expression contributed to tapetum PCD for releasing the fertile pollen. Glyma.U045200 showed a lower methylation level in NJCMS5A compared with NJCMS5B, which may promote the expression of gene Glyma.U045200 in NJCMS5A, and eventually affected normal tapetum PCD, leading to pollen abortion.

\section{Transcription factor (TF) potentially related to CMS}

In plant, TF plays a critical role in the regulation of plant metabolism and development by modifying the expression of their target genes [46]. For example, R2R3-type $M Y B$ gene could control many aspects of plant in secondary metabolism, as well as regulation of plant cell fate and identity [47]. MYB98, a member of the R2R3MYB family TF, mainly regulates of transcriptional events in the synergid cell [48]. So it is necessary for pollen tube guidance and successful fertilization in flowering plant [49]. In the mature pollen of Arabidopsis, the expression of MYB97 was much higher than normal, and a triple mutant of myb97-1, myb101-2 and myb120-3 caused overgrowth of the pollen tube into the embryo sac and disrupted sperm cell discharge, leading the failure of fertilization [50]. In this study, the DMG Glyma.U028100 was predicted to encode a homolog of MYB98, and Glyma.08G317600 was also predicted to encode a MYB TF protein. The promoter region of both DMGs showed a lower methylation levels in NJCMA5A compared with NJCMS5B, which implied that the abnormal expression of these two genes may disorder the development of floral organ, resulting in male sterility in NJCMS5A.

The MADS-box TF family is widespread in plant and animal, which involves in diverse and important biological functions [51], especially in floral organ process. Recently, soybean GmMADS28 which encodes a MADSbox protein has been reported [52]. The genetically modified anther of 35S:GmMADS28 was not dehiscent and failed to release pollen, which largely led to plant male sterility [52]. In this study, the DMG Glyma.U040000 was annotated as an AP2/B3-like TF family protein contained a B3 domain. In Arabidopsis, VRN1, 
Table 3 Significant Gene Ontology (GO) terms of hyper-methylated genes in NJCMS5A

\begin{tabular}{|c|c|c|c|c|c|}
\hline GO accession & Term type & Description & DMR_item & $\mathrm{BG}_{\text {_ item }}$ & $P_{\text {_ value }}$ \\
\hline GO:0019058 & $\mathrm{BP}$ & viral infectious cycle & 6 & 364 & 0.0022802 \\
\hline GO:0006011 & $\mathrm{BP}$ & UDP-glucose metabolic process & 1 & 1 & 0.0036546 \\
\hline GO:0015977 & $\mathrm{BP}$ & carbon fixation & 2 & 25 & 0.0037648 \\
\hline GO:0016042 & $\mathrm{BP}$ & lipid catabolic process & 3 & 109 & 0.0075620 \\
\hline GO:0044403 & $\mathrm{BP}$ & symbiosis, encompassing mutualism through parasitism & 8 & 789 & 0.0086707 \\
\hline GO:0071704 & $\mathrm{BP}$ & organic substance metabolic process & 76 & 16,860 & 0.0089794 \\
\hline GO:0044419 & $\mathrm{BP}$ & interspecies interaction between organisms & 8 & 796 & 0.0091147 \\
\hline GO:0006099 & $\mathrm{BP}$ & tricarboxylic acid cycle & 2 & 40 & 0.0094446 \\
\hline GO:0000723 & $\mathrm{BP}$ & telomere maintenance & 5 & 350 & 0.0094633 \\
\hline GO:0032200 & $\mathrm{BP}$ & telomere organization & 5 & 350 & 0.0094633 \\
\hline GO:0060249 & $\mathrm{BP}$ & anatomical structure homeostasis & 5 & 350 & 0.0094633 \\
\hline GO:0019067 & $\mathrm{BP}$ & viral assembly, maturation, egress, and release & 3 & 130 & 0.0121930 \\
\hline GO:0009060 & $\mathrm{BP}$ & aerobic respiration & 2 & 46 & 0.0123550 \\
\hline GO:0016032 & $\mathrm{BP}$ & viral process & 7 & 725 & 0.0175200 \\
\hline GO:0044238 & $\mathrm{BP}$ & primary metabolic process & 72 & 16,245 & 0.0190260 \\
\hline GO:0009225 & $\mathrm{BP}$ & nucleotide-sugar metabolic process & 1 & 6 & 0.0217300 \\
\hline GO:0034219 & $\mathrm{BP}$ & carbohydrate transmembrane transport & 1 & 6 & 0.0217300 \\
\hline GO:0071806 & $\mathrm{BP}$ & protein transmembrane transport & 2 & 66 & 0.0244210 \\
\hline GO:0006367 & $\mathrm{BP}$ & transcription initiation from RNA polymerase II promoter & 2 & 67 & 0.0251130 \\
\hline GO:0030150 & $\mathrm{BP}$ & protein import into mitochondrial matrix & 1 & 7 & 0.0253060 \\
\hline GO:0044764 & $\mathrm{BP}$ & multi-organism cellular process & 7 & 785 & 0.0256590 \\
\hline GO:0015074 & $\mathrm{BP}$ & DNA integration & 2 & 69 & 0.0265210 \\
\hline GO:0044237 & $\mathrm{BP}$ & cellular metabolic process & 67 & 15,179 & 0.0286360 \\
\hline GO:0072519 & $\mathrm{BP}$ & parasitism & 1 & 9 & 0.0324180 \\
\hline GO:0018149 & $\mathrm{BP}$ & peptide cross-linking & 1 & 10 & 0.0359550 \\
\hline GO:0006464 & $\mathrm{BP}$ & cellular protein modification process & 19 & 3357 & 0.0370220 \\
\hline GO:0036211 & $\mathrm{BP}$ & protein modification process & 19 & 3357 & 0.0370220 \\
\hline GO:0006468 & $\mathrm{BP}$ & protein phosphorylation & 15 & 2522 & 0.0427720 \\
\hline GO:0019068 & $\mathrm{BP}$ & virion assembly & 2 & 90 & 0.0430940 \\
\hline GO:0043170 & $\mathrm{BP}$ & macromolecule metabolic process & 57 & 12,853 & 0.0449750 \\
\hline GO:0043412 & $\mathrm{BP}$ & macromolecule modification & 20 & 3665 & 0.0451580 \\
\hline GO:0032940 & $\mathrm{BP}$ & secretion by cell & 3 & 221 & 0.0477090 \\
\hline GO:0046903 & $\mathrm{BP}$ & secretion & 3 & 221 & 0.0477090 \\
\hline GO:0006807 & $\mathrm{BP}$ & nitrogen compound metabolic process & 41 & 8794 & 0.0487850 \\
\hline GO:0000079 & $\mathrm{BP}$ & $\begin{array}{l}\text { regulation of cyclin-dependent protein serine/threonine } \\
\text { kinase activity }\end{array}$ & 1 & 14 & 0.0499750 \\
\hline GO:0071900 & $\mathrm{BP}$ & regulation of protein serine/threonine kinase activity & 1 & 14 & 0.0499750 \\
\hline GO:0016592 & CC & mediator complex & 4 & 183 & 0.0046636 \\
\hline GO:0036126 & $\mathrm{CC}$ & sperm flagellum & 1 & 2 & 0.0072960 \\
\hline GO:0097223 & $\mathrm{CC}$ & sperm part & 1 & 2 & 0.0072960 \\
\hline GO:0097228 & $\mathrm{CC}$ & sperm principal piece & 1 & 2 & 0.0072960 \\
\hline GO:0005654 & CC & nucleoplasm & 5 & 384 & 0.0136830 \\
\hline GO:0044451 & $\mathrm{CC}$ & nucleoplasm part & 5 & 384 & 0.0136830 \\
\hline GO:0071203 & $\mathrm{CC}$ & WASH complex & 2 & 61 & 0.0210820 \\
\hline
\end{tabular}


Table 3 Significant Gene Ontology (GO) terms of hyper-methylated genes in NJCMS5A (Continued)

\begin{tabular}{|c|c|c|c|c|c|}
\hline GO:0033644 & $\mathrm{CC}$ & host cell membrane & 2 & 67 & 0.0251130 \\
\hline GO:0044218 & $\mathrm{CC}$ & other organism cell membrane & 2 & 67 & 0.0251130 \\
\hline GO:0044279 & CC & other organism membrane & 2 & 67 & 0.0251130 \\
\hline GO:0044441 & $\mathrm{CC}$ & cilium part & 1 & 7 & 0.0253060 \\
\hline GO:0036128 & CC & catSper complex & 1 & 9 & 0.0324180 \\
\hline GO:0030658 & $\mathrm{CC}$ & transport vesicle membrane & 2 & 87 & 0.0405380 \\
\hline GO:0019031 & CC & viral envelope & 4 & 354 & 0.0413760 \\
\hline GO:0036338 & CC & viral membrane & 4 & 354 & 0.0413760 \\
\hline GO:0005891 & CC & voltage-gated calcium channel complex & 1 & 13 & 0.0464890 \\
\hline GO:0034704 & $\mathrm{CC}$ & calcium channel complex & 1 & 13 & 0.0464890 \\
\hline GO:0004675 & MF & transmembrane receptor protein serine/threonine kinase activity & 2 & 18 & 0.0019525 \\
\hline GO:0008964 & MF & phosphoenolpyruvate carboxylase activity & 2 & 20 & 0.0024130 \\
\hline GO:0008234 & MF & cysteine-type peptidase activity & 5 & 273 & 0.0033820 \\
\hline GO:0019199 & MF & transmembrane receptor protein kinase activity & 2 & 25 & 0.0037648 \\
\hline GO:0001076 & MF & $\begin{array}{l}\text { RNA polymerase II transcription factor binding transcription factor } \\
\text { activity }\end{array}$ & 4 & 180 & 0.0043995 \\
\hline GO:0001104 & MF & RNA polymerase II transcription cofactor activity & 4 & 180 & 0.0043995 \\
\hline GO:0017111 & MF & nucleoside-triphosphatase activity & 17 & 2371 & 0.0059866 \\
\hline GO:0000988 & MF & protein binding transcription factor activity & 5 & 320 & 0.0065718 \\
\hline GO:0004611 & MF & phosphoenolpyruvate carboxykinase activity & 2 & 34 & 0.0068906 \\
\hline GO:0016462 & MF & pyrophosphatase activity & 17 & 2410 & 0.0070057 \\
\hline GO:0004197 & MF & cysteine-type endopeptidase activity & 4 & 221 & 0.0089745 \\
\hline GO:0016818 & MF & $\begin{array}{l}\text { hydrolase activity, acting on acid anhydrides, in phosphorus-containing } \\
\text { anhydrides }\end{array}$ & 17 & 2498 & 0.0098272 \\
\hline GO:0000989 & MF & transcription factor binding transcription factor activity & 4 & 228 & 0.0099793 \\
\hline GO:0003712 & MF & transcription cofactor activity & 4 & 228 & 0.0099793 \\
\hline GO:0016787 & MF & hydrolase activity & 38 & 7372 & 0.0141010 \\
\hline GO:0016817 & MF & hydrolase activity, acting on acid anhydrides & 17 & 2626 & 0.0154860 \\
\hline GO:0005516 & MF & calmodulin binding & 2 & 56 & 0.0179520 \\
\hline GO:0000149 & MF & SNARE binding & 1 & 5 & 0.0181410 \\
\hline GO:0019905 & MF & syntaxin binding & 1 & 5 & 0.0181410 \\
\hline GO:1,901,363 & MF & heterocyclic compound binding & 59 & 12,823 & 0.0194820 \\
\hline GO:0097159 & MF & organic cyclic compound binding & 59 & 12,831 & 0.0197370 \\
\hline GO:0004386 & MF & helicase activity & 9 & 1116 & 0.0217290 \\
\hline GO:0004308 & MF & exo-alpha-sialidase activity & 1 & 6 & 0.0217300 \\
\hline GO:0004683 & MF & calmodulin-dependent protein kinase activity & 1 & 6 & 0.0217300 \\
\hline GO:0016997 & MF & alpha-sialidase activity & 1 & 6 & 0.0217300 \\
\hline GO:0034062 & MF & RNA polymerase activity & 5 & 448 & 0.0247360 \\
\hline GO:0070403 & MF & NAD+ binding & 1 & 7 & 0.0253060 \\
\hline GO:0016779 & MF & nucleotidyltransferase activity & 7 & 785 & 0.0256590 \\
\hline GO:0003676 & MF & nucleic acid binding & 33 & 6489 & 0.0271790 \\
\hline GO:0003852 & MF & 2-isopropylmalate synthase activity & 1 & 8 & 0.0288680 \\
\hline GO:0008083 & MF & growth factor activity & 2 & 73 & 0.0294300 \\
\hline GO:0003899 & MF & DNA-directed RNA polymerase activity & 4 & 319 & 0.0299720 \\
\hline GO:0003677 & MF & DNA binding & 23 & 4224 & 0.0332160 \\
\hline GO:0016298 & MF & lipase activity & 3 & 197 & 0.0359010 \\
\hline
\end{tabular}


Table 3 Significant Gene Ontology (GO) terms of hyper-methylated genes in NJCMS5A (Continued)

\begin{tabular}{|c|c|c|c|c|c|}
\hline GO:0004325 & MF & ferrochelatase activity & 1 & 10 & 0.0359550 \\
\hline GO:0038023 & MF & signaling receptor activity & 4 & 347 & 0.0389240 \\
\hline GO:0047750 & MF & cholestenol delta-isomerase activity & 1 & 11 & 0.0394790 \\
\hline GO:0003678 & MF & DNA helicase activity & 6 & 693 & 0.0423700 \\
\hline GO:0016863 & MF & $\begin{array}{l}\text { intramolecular oxidoreductase activity, transposing } \\
\mathrm{C}=\mathrm{C} \text { bonds }\end{array}$ & 1 & 12 & 0.0429910 \\
\hline GO:0046915 & MF & transition metal ion transmembrane transporter activity & 3 & 221 & 0.0477090 \\
\hline GO:0046789 & MF & host cell surface receptor binding & 1 & 14 & 0.0499750 \\
\hline
\end{tabular}

BP biological process; CC cellular component; $M F$ molecular functional; DMR differentially methylated region; $B G$ : background

as a member of the REM subfamily, contained two B3 DNA-binding domains. It could interact with AGAMOUS-like 20 (AGL20), flowering locus $\mathrm{T}$ (FT) and MADS-box protein flowering locus C (FLC), as well as with other regulators to restrain flowering [53]. The promoter region of Glyma.U040000 with abnormal methylation level in NJCMS5A was supposed to negatively regulate gene expression, which may indirectly influence flower structure and flowering mechanism, leading to male sterility in NJCMS5A.

\section{DMGs encoding mitochondrial protein potentially related to CMS}

Recently, it has also been proposed that the fertility of CMS plant could be restored by corresponding nuclear fertility restoration (RF) gene. And RF genes that were encoded by pentatricopeptide repeat (PPR) protein have been isolated from rice, carrot, and pepper [54-56]. RNA processing factor 1 gene (RPF1) belongs to a subgroup of PPR protein, which could guide RF gene product to restore CMS. In this study, the DMGs Glyma.14G212600 and Glyma.16G195100 were placed in the mitochondria. Glyma.16G195100 was homologous with RPF1, which was supposed to play a role in fertility recovery in CMS plant [57]. In addition, the promoter of Glyma.14G212600 was hyper-methylated in NJCMS5A compared with NJCMS5B, implying the expression of Glyma.14G212600 may be suppressed. So, Glyma.14G212600 and Glyma.16G195100 were predicted to encode two PPR proteins, which may be associated with editing mitochondrial gene to impact the expression of the CMS-related genes in the mitochondria, resulting in CMS in NJCMS5A.

\section{Conclusion}

In the study, the genome-wide methylation profiles of the soybean CMS line NJCMS5A and its maintainer NJCMS5B were obtained from whole-genome bisulfite sequencing. As a result, 739 DMRs and 485 DMGs, including 353 high-credible methylated genes, 56 methylated genes coding unknown protein and 76 novel methylated genes with no known function were identified. In addition, those valid methylated genes were identified as 281 hypo-methylated genes and 88 hypermethylated genes in NJCMS5A relative to NJCMS5B. According to the gene region annotations, gene function, GO and KEGG pathway analysis, as well as the previous articles reported studies of male sterility in the plant, 8 key DMGs that may be related to soybean CMS were discussed, which mainly involved in participating in pollen development, encoding TF and the mitochondrial genome. This study provides DNA methylome of

Table 4 Identified eight genes potentially related to cytoplasmic male sterility (CMS)

\begin{tabular}{lllll}
\hline NO. & Gene ID & DMR region & $\begin{array}{l}\text { Methylation status } \\
\text { (NJCMS5A vs. NJCMS5B) }\end{array}$ & Description \\
\hline 1 & Glyma.U015500 & promoter & Hypo & bHLH DNA-binding super family protein \\
2 & Glyma.16G195100 & gene body & Hypo & mitochondrial mRNA modification \\
3 & Glyma.06G248800 & gene body & Hypo & ABC-2 type transporter family protein \\
4 & Glyma.U045200 & gene body & Hypo & cysteine proteinases super family protein \\
5 & Gromoter & & MYB domain protein 97 \\
6 & Glyma.08G317600 & promoter & Hypo & MYB domain protein 98 \\
7 & Glyma.U040000 & promoter & Hypo & AP2/B3-like transcriptional factor family protein \\
8 & Glyma.14G212600 & promoter & Hyper & PPR super family protein
\end{tabular}

Hyper DNA methylation level in NJCMS5A was higher than that in NJCMS5B; Hypo DNA methylation level in NJCMS5A was lower than that in NJCMS5B 

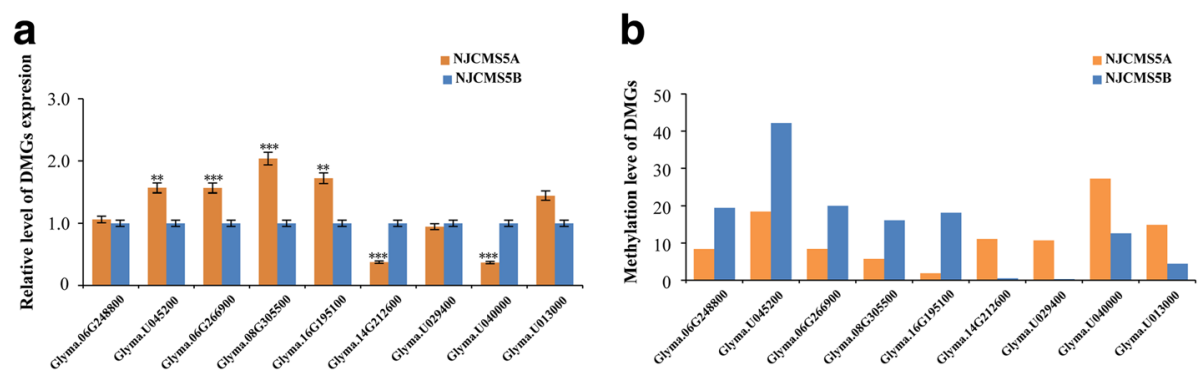

Fig. 8 Expression level of nine DMGs validated by qRT-PCR. a qRT-PCR analysis of nine DMGs in NJCMS5A and NJCMS5B. X-axis represented gene name, the red column represented NJCMS5A, and blue column represented CK (NJCMS5B); Y-axis represented the relative level of gene expression. All. qRT-PCR reactions were performed with three biological replicates; $\boldsymbol{b}$ DNA methylation level of nine DMGs in NJCMS5A and NJCMS5B

soybean CMS line and its maintainer line for the first time, which will contribute to further understanding the methylation mechanism in soybean CMS.

\section{Methods}

\section{Plant materials}

The soybean [Glycine $\max$ (L.) Merr.] CMS line NJCMS5A was developed through consecutive backcross procedures with NJCMS1A [26-28] as donor parent and Wandou28 (designated as NJCMS5B afterwards) as the recurrent parent. Both NJCMS5A and NJCMS5B were planted in the summer of 2014 at the Jiangpu Experimental Station, National Center for Soybean Improvement, Nanjing Agricultural University, Nanjing, Jiangsu, China. The male-sterile plant was identified through three kinds of methods including the dehiscence of anthers, germination rate of pollen, and performance of plant at maturity. Because it is very difficult to judge the precise development stage of pollen from the appearance of the flower buds in soybean, so during flowering period, the flower buds in different sizes were collected and pooled from NJCMS5A and NJCMS5B plant respectively, then immediately frozen in liquid nitrogen and stored at $-80{ }^{\circ} \mathrm{C}$ for further use.

\section{Total DNA extraction and DNA library construction}

Genomic DNA was extracted from the flower bud of NJCMS5A and NJCMS5B using DNeasy Plant Mini Kit (Qiagen Valencia, CA, USA), respectively, according to the manufacturer's instruction. First, the purity of DNA sample was detected using the NanoPhotometer Spectrophotometer (Implen, CA, USA), and the concentration of DNA sample was measured using UV-Vis Spectrophotometer (Thermo Scientific, MA, USA). Subsequently, DNA sample was randomly sonicated to $200-300$ bp using Covaris S220 (Thermo Scientific, MA, USA). After end repair and adenylation, the sonicated DNA fragments were ligated to cytosine-methylated barcodes according to manufacturer's instruction. DNA fragment was treated twice with bisulfite using EZ DNA Methylation-Gold Kit
(Zymo Research, CA, USA). After that, DNA library was constructed by PCR amplification. DNA library concentration was quantified by Qubit2.0 Fluorometer (Life Technologies, CA, USA), and the size of the insert fragment was tested by Biological Analyzer Agilent 2100 (Agilent, CA, USA). Finally, the qualified DNA library was sequenced on an Illumina Hiseq 2500 platform with paired-end reads by Novogene Bioinformatics Technology Co.Ltd. (Beijing, China).

\section{Bioinformatics analysis of DNA methylation sequencing data}

After sequencing, the raw reads were filtered to remove adapters, Ns and low quality reads. The remaining reads called clean reads were stored in FASTQ format. Clean reads were aligned to the reference genome of soybean (https://phytozome.jgi.doe.gov/pz/portal.html\#!info?alias=Org_Gmax) using Bismark software (version 0.12.5) with the default parameters [58]. The genetic structure annotation file was obtained from the public fit site of Phytozome (https://phytozome.jgi.doe.gov/pz/portal.html\#!info?alias=Org_Gmax). Annotations of transposable element (TE) were downloaded from the SoyTEdb database (http://www.soybase.org/soytedb/) and TEs were plotted in $20 \mathrm{~kb}$ windows along the chromosome. Finally, only reads that were uniquely mapped to soybean reference genome were used for further analysis. $\mathrm{mC}$ percent (\%) was calculated as the percentage of methylated $\mathrm{C}$ sites in the whole genome. mCG percent (\%), $\mathrm{mCHG}$ percent (\%) and $\mathrm{mCHH}$ percent (\%) were calculated as the percentage of methylated $\mathrm{C}$ site in the $\mathrm{C}, \mathrm{CG}$ and $\mathrm{CHH}$ contexts, respectively.

To identify the methylation site, we modeled the sum $\boldsymbol{s}_{\mathbf{i}, \mathbf{j}}^{+}$of methylated counts as a binomial (Bin) random variable with methylation rate $\mathbf{r}_{\mathbf{i}, \mathbf{j}}, \boldsymbol{s}_{\mathbf{i}, \mathbf{j}}^{+} \sim \operatorname{Bin}\left(\boldsymbol{s}_{\mathbf{i}, \mathbf{j}}^{+}+\boldsymbol{s}_{\mathbf{i}, \mathbf{j}}^{-}, \mathbf{r}_{\mathbf{i}, \mathbf{j}}\right)$. Then we employed a sliding-window approach, which is conceptually similar to the approaches used for bulk BSSeq (http://www.bioconductor.org/packages/2.13/bioc/ $\mathrm{html} / \mathrm{bsseq} \cdot \mathrm{html}$ ). With window size $\mathrm{w}=3000 \mathrm{bp}$, step size $=600 \mathrm{bp}[59]$ and sequencing depth $\geq 5, q$-value 
$<0.05$, the sum of methylated and unmethylated counts in every window were calculated. Else, the methylation level (ML) for each C site was defined as $M L(C)=$ reads $(\mathbf{m C}) /$ reads $(\mathbf{m C}+\mathbf{u m C})$. The calculated ML was further corrected using with non-conversion rate r, $M L$ (corrected $)=(M L-r) /(1-r)$ [60]. Density was calculated by sites $(\mathrm{mC}) /$ sites $(\mathrm{mC}+\mathrm{umC})$. $\mathrm{ML}$ and density were both used for the analysis of the reads distribution in soybean chromosome and in the different genome functional region (promoter, 5'UTR, exon, intron, and 3'UTR) under three different contexts (CG, CHG and $\mathrm{CHH}$ ).

Differentially methylated regions (DMRs) between NJCMS5A and NJCMS5B were identified using the swDMR software (https://sourceforge.net/projects/ swdmr/) with a sliding-window approach [61]. The window is set to $1000 \mathrm{bp}$ and step length is $100 \mathrm{bp}$. Fisher test is used to detect the DMRs with "FDR $\leq 0.05$ and coverage changes $\geq 5$ ". Then the differentially methylated genes (DMGs) were collected from DMRs that overlapped gene functional region (promoter, 5'UTR, exon, intron and 3'UTR) with at least $1 \mathrm{bp}$. Next, Gene Ontology (GO) enrichment analysis (http://www.geneontology.org/) was performed for all the identified DMGs using the GOseq R package $(P$-value $<0.05)$ [62]. Finally, the hypergeometric test statistical method was used for the metabolic pathway analysis of all identified DMGs in Kyoto Encyclopedia of Genes and Genomes (KEGG) pathways (http://www.genome.jp/kegg/) using KOBAS software [63].

\section{Validation of the sequencing data by bisulfite treatment} Bisulfite genomic sequencing is regarded as a goldstandard for detection of DNA methylation, because it provides a qualitative and quantitative method to apply to a limit number of loci [64]. Twenty-five DMRs were selected randomly to validate the reliability of the sequencing data using a nest PCR (nPCR). All the primers (Additional file 10) were designed using the website of Kismeth (http://katahdin.mssm.edu/kismeth/revpage.pl), and synthesized commercially (Invitrogen, Shanghai, China). Briefly, $1 \mu \mathrm{g}$ of genomic DNA was treated by bisulfite according to the protocol of the EZ DNA Methylation-Gold Kit (Zymo Research, CA, USA), and used as the template for the following nPCR amplification. Then, the bisulfite treatment PCR (BS-PCR) was performed in a $50 \mu \mathrm{l}$ reaction mixture, containing $25 \mu \mathrm{l}$ premix EX Taq DNA polymerase (TaKaRa, Osaka, Japan), $25 \mu \mathrm{g}$ bisulfite-treated DNA and $0.2 \mu \mathrm{M}$ of each pair of primers with a nest PCR. Next, products were purified using Gel Extraction Kit (Axygen, CA, USA), and cloned into the pMD19-T Simple Vector (TaKaRa, Osaka, Japan). Each DMR amplified with 10-14 clones was sequenced by Invitrogen Trading Shanghai Co.Ltd. (Shanghai, China), and the sequencing results were spliced and edited by BioEdit and vector NTI8 software, then analyzed in the Kismeth website.

\section{Validation of candidate DMGs by quantitative real-time PCR (qRT-PCR)}

qRT-PCR was used to verify the methylated gene expression. All the primers (Additional file 11) were designed by Primer-BLAST, and synthesized commercially (Invitrogen, Shanghai, China). Firstly, $1 \mu \mathrm{g}$ of total RNA was reverse-transcribed by reverse transcriptase according to the protocol of iScript cDNA Synthesis Kit (Bio-Rad, CA, USA), and used as the template for the following qRT-PCR amplification. Then, qRT-PCR was performed on the Bio-Rad CFX96 Touch q-PCR System (Bio-Rad, CA, USA) with iTaq Universal SYBR Green Super mix (Bio-Rad, CA, USA). Each reaction was replicated three times, and $\beta$-actin gene was used as the internal control gene. The relative level of gene expression was evaluated by the $2^{-\Delta \Delta C t}$ method, NJCMS5B served as the control. The relative level of gene expression greater than 1 was regarded as up-regulated and less than 1 was regarded as down-regulated. Student's $t$-test was adopted to test the difference between the relative level of gene expression of NJCMS5A and the control NJCMS5B. The means of $2^{-\Delta \Delta \mathrm{Ct}}$ were considered significantly different at $P<0.05$.

Differential expression analysis of mRNA sequencing data All the mRNA-seq analysis was based on the clean data with high quality. The expression quantity of each gene (fragments per kilobase of exon model per million mapped fragments, FPKM) was estimated by Cuffdiff software [65]. "FDR (False Discovery Rate) $\leq 0.05$ [66, 67] and $\mid \log 2 \mathrm{FC}$ (Fold Change) $\mid \geq 1$ " were used as the threshold for judging the significant of gene expression difference.

\section{Additional files}

Additional file 1: Data analysis of soybean genome-wide methylation sequencing. (PDF $207 \mathrm{~kb}$ )

Additional file 2: Density distribution of genome-wide methylation in soybean chromosome. (PDF $1510 \mathrm{~kb}$ )

Additional file 3: Gene expression level of transcriptome data. (XLS $8687 \mathrm{~kb})$

Additional file 4: Graph of gene expression level in gene functional region. (PDF $146 \mathrm{~kb}$ )

Additional file 5: Number of differentially methylated regions (DMRs) between NJCMS5A and NJCMS5B. (XLS 695 kb)

Additional file 6: Number of differentially methylated genes (DMGs) between NJCMS5A and NJCMS5B. (XLS $165 \mathrm{~kb}$ )

Additional file 7: Result of target DMRs in the $\mathrm{CG}, \mathrm{CHG}$ and $\mathrm{CHH}$ context. (DOCX $22 \mathrm{~kb}$ )

Additional file 8: Validation of sequencing data by bisulfite treatment. (DOCX $16 \mathrm{~kb}$ ) 
Additional file 9: DMGs predicted to be enriched in KEGG pathways. (DOCX $18 \mathrm{~kb}$ )

Additional file 10: Primer pairs used for bisulfite treatment. (DOCX $21 \mathrm{~kb}$ )

Additional file 11: Primer pairs used for quantitative real-time $P C R$ (qRT-PCR). (DOCX $17 \mathrm{~kb}$ )

\section{Abbreviations}

bHLH: basic helix-loop-helix; BP: biological process; CC: cellular component: CMS: cytoplasmic male sterility; DMG: differentially methylated gene; DMR: differentially methylated region; DNMT: DNA methyltransferase; DYT1: dysfunctional tapetum 1; GO: Gene Ontology; KEGG: Kyoto Encyclopedia of Genes and Genomes; mC: methylated cytosine; MF: molecular function; PCD: programmed cell death; PPR: pentatricopeptide repeat-containing; qRT-PCR: quantitative real-time PCR; RF: fertility restoration; RPF1: RNA processing factor 1; TE: transposable element; TF: transcription factor; WGBS: whole-genome bisulfite sequencing

\section{Acknowledgements}

We would thank Novogene Bioinformatics Technology Co.Ltd. (Beijing, China) for conducting the whole-genome methylation sequencing.

\section{Funding}

This work was supported by the National Key R\&D Program of China (2016YFD0101500, 2016YFD0101504), the National Hightech R\&D Program of China (2011AA10A105), and the Program for Changjiang Scholars and Innovative Research Team in University (PCSIRT13073).

\section{Availability of data and materials}

All whole-genome bisulfite sequencing raw data were deposited in the National Center for Biotechnology Information (NCBI) under the accession number GSE101652 (http://www.ncbi.nlm.nih.gov/geo/query/ acc.cgi?acc=GSE101652). The transcriptome sequencing data were unpublished.

\section{Authors' contributions}

SPY and YWL conceived and designed the experiments. YWL performed the experiments and analyzed the data. YWL, XLD, XW, TTH, HZ, LSY, TLW and LFC contributed reagents/materials/analysis tools. YWL and SPY wrote the paper. SPY, JYG and YWL revised the paper. All authors read and approved the final manuscript.

\section{Authors' information}

All authors came from Soybean Research Institute, National Center for Soybean Improvement, Key Laboratory of Biology and Genetic Improvement of Soybean (General, Ministry of Agriculture), State Key Laboratory of Crop Genetics and Germplasm Enhancement, Jiangsu Collaborative Innovation Center for Modern Crop Production, Nanjing Agricultural University, Nanjing 210095, China.

\section{Ethics approval and consent to participate}

The plant materials came from Soybean Research Institute, National Center for Soybean Improvement, Key Laboratory of Biology and Genetic Improvement of Soybean (General, Ministry of Agriculture), Nanjing Agricultural University, Nanjing 210095, China. The use of plant materials complied by the ethics of the People's Republic of China.

\section{Consent for publication}

Not applicable.

\section{Competing interests}

The authors declared that they had no competing interests.

\section{Publisher's Note}

Springer Nature remains neutral with regard to jurisdictional claims in published maps and institutional affiliations.
Received: 24 March 2017 Accepted: 25 July 2017

Published online: 10 August 2017

\section{References}

1. Hanson MR. Plant mitochondrial mutations and male sterility. Annu Rev Genet. 1991;25:461-486

2. Chen L, Liu YG. Male sterility and fertility restoration in crops. Annu Rev Plant Biol. 2014;65:579-606.

3. Carlsson J, Glimelius K. Cytoplasmic male-sterility and nuclear encoded fertility restoration. Plant Biol. 2010;1:469-91.

4. Hu J, Wang K, Huang WC, Liu G, Gao Y, Wang JM, et al. The rice pentatricopeptide repeat protein RF5 restores fertility in Hong-Lian cytoplasmic male-sterile lines via a complex with the glycine-rich protein GRP162. Plant Cell. 2012;24(1):109-122.

5. Li JJ, Han SH, Ding XL, He TT, Dai JY, Yang SP, et al. Comparative transcriptome analysis between the cytoplasmic male sterile line NJCMS1A and its maintainer NJCMS1B in soybean (Glycine max (L.) Merr.). PloS One. 2015;10(5):e0126771

6. Li JJ, Ding XL, Han SH, He TT, Zhang H, Yang LS, et al. Differential proteomics analysis to identify proteins and pathways associated with male sterility of soybean using iTRAQ-based strategy. J Proteome. 2016;138:72-82.

7. Ding XL, Li JJ, Zhang H, He TT, Han SH, Li YW, et al. Identification of miRNAs and their targets by high-throughput sequencing and degradome analysis in cytoplasmic male-sterile line NJCMS1A and its maintainer NJCMS1B of soybean. BMC Genomics. 2016;17:24.

8. Chang SX, Wang YK, Lu JJ, Gai JY, Li JJ, Chu P, et al. The mitochondrial genome of soybean reveals complex genome structures and gene evolution at intercellular and phylogenetic levels. PLoS One. 2013;8(2): e56502.

9. Cao R, Wang LJ, Wang HB, Xia L, Zhang $Y$, et al. Role of histone H3 lysine 27 methylation in polycomb-group silencing. Science. 2002;298(5595):1039-43.

10. Ding JH, Shen JQ, Mao HL, Xie WB, Li XH, Zhang QF. RNA-directed DNA methylation is involved in regulating photoperiod-sensitive male sterility in rice. Mol Plant. 2012;5(6):1210-1216

11. Schmitz RJ, Sung S, Amasino RM. Histone arginine methylation is required for vernalization-induced epigenetic silencing of FLC in winter-annual Arabidopsis thaliana. Proc Natl Acad Sci USA. 2008:105(2):411-16.

12. Takuno S, Gaut BS. Gene body methylation is conserved between plant orthodoxs and is of evolutionary consequence. Proc Natl Acad Sci USA 2013;110(5):1797-1802

13. Finnegan EJ, Peacock WJ, Dennis ES. DNA methylation, a key regulator ofplant development and other processes. Curr Opin Genet Dev. 2000;10(2): 217-223.

14. Song QX, Lu X, Li QT, Chen H, Hu XY, Ma B, et al. Genome-wide analysis of DNA methylation in soybean. Mol Plant. 2013;6(6):1961-74.

15. Zhang X, Yazaki J, Sundaresan A, Cokus S, Chan SW, Chen H, et al. Genomewide high-resolution mapping and functional analysis of DNA methylation in Arabidopsis. Cell. 2006;126(6):1189-1201.

16. Cao X, Aufsatz W, Zilberman D, Mette MF, Huang MS, et al. Role of the DRM and CMT3 methyltransferases in RNA-directed DNA methylation. Curr Biol. 2003;13(24):2212-17.

17. Henderson IR, Deleris A, Wong W, Zhong X, Chin HG, Horwitz GA, et al. The de novo cytosine methyltransferase DRM2 requires intact UBA domains and a catalytically mutated paralog DRM3 during RNA-directed DNA methylation in Arabidopsis thaliana. PLOS Genet. 2010:6(10):e1001182.

18. Dalakouras A, Moser M, Zwiebel M, Krczal G, Hell R, Wassenegger M Ahairpin RNA construct residing in an intron efficiently triggered RNAdirected DNA methylation in tobacco. Plant J. 2009;60(5):840-51.

19. Arteagavazquez M, Sidorenko L, Rabanal FA, Shrivistava R, Nobuta K, Green PJ, et al. RNA-mediated trans-communication can establish paramutation at the b1 locus in maize. Proc Natl Acad Sci USA. 2010;107(29):12986-991.

20. Wu T, Yuan T, Tsai SN, Wang C, Sun SM, Lam HM, et al. Mass spectrometry analysis of the variants of histone $\mathrm{H} 3$ and $\mathrm{H} 4$ of soybean and their posttranslational modifications. BMC Plant Biol. 2009:9:98.

21. Yan HH, Kikuchi SJ, Neumann P, Zhang WL, Wu YF, Chen F, et al. Genomewide mapping of cytosine methylation revealed dynamic DNA methylation patterns associated with genes and centromeres in rice. Plant J. 2010;63(3): 353-65.

22. Zhao YL, Yu SX, Ye WW, Wang HM, Wang JJ, Fang BX. Study on DNA cytosine methylation of cotton (Gossypium hirsutum L.) genome and its implication for salt tolerance. Agric Sci China. 2010;09(6):783-791. 
23. Chen XJ, Hu JH, Zhang HY, Ding Y. DNA methylation changes in photoperiod-thermo-sensitive male sterile rice PA64S under two different conditions. Gene. 2014;537(1):143-8.

24. Hu JH, Chen XJ, Zhang HY, Ding Y. Genome-wide analysis of DNA methylation in photoperiod- and thermo-sensitive male sterile rice Peiai 64S. BMC Genomics. 2015:16:102

25. Lu YL, Liu Y, Wang J, Cao M, Rong TZ. Variation and patterns of DNA methylation in maize C-type CMS lines and their maintainers. J Plant Biochem Biotechnol. 2009;19(1):43-50.

26. Gai JY, Cui ZL, Ji DF, Ren ZJ, Ding DR. A report on the nuclear cytoplasmic male sterility from a cross between two soybean cultivars. Soy Genet Newsl. 1995;22:55-58.

27. Ding DR, Gai JY, Cui ZL, Yang SP, Qiu JX. Development and verification of the cytoplasmic-nuclear male sterile soybean line NJCMS1A and its maintainer NJCMS1B. Chin Sci Bull. 1999:44(2):191-192.

28. Gai JY, Ding DR, Cui ZL, Qiu JX. Development and performance of the cytoplasmic-nuclear male sterile line NJCMS1A of soybean. Sci Agric Sin. 2000;(1):41-47

29. Schmitz RJ, He YP, Khan SM, Joshi T, Urich MA, et al. Epigenome-wide inheritance of cytosine methylation variants in a recombinant inbred population. Genome Res. 2013;23(10):1663-74.

30. Du J, Grant D, Tian Z, Nelson RT, Zhu L, Shoemaker RC, et al. SoyTEdb: a comprehensive database of transposable elements in the soybean genome. BMC Genomics. 2010;11:113.

31. Schmutz J, Cannon SB, Schlueter J, Ma J, Mitros T, Nelson W, et al. Genome sequence of the palaeopolyploid soybean. Nature. 2010;463(7278):178-83.

32. Xu J, Yang CY, Yuan Z, Zhang DS, Gondwe MY, Ding Z, et al. Aborted microspores regulatory network is required for postmeiotic male reproductive development in Arabidopsis thaliana. Plant Cell. 2010;22(1):91-107.

33. Scott RJ, Spielman M, Dickinson HG. Stamen structure and function. Plant Cell. 2004;16(Suppl):S46-60.

34. Wilson ZA, Morroll SM, Dawson J, Swarup R, Tighe PJ. The Arabidopsis male sterility1 ( MS1) gene is a transcriptional regulator of male gametogenesis, with homology to the PHD-finger family of transcription factors. Plant J. 2001;28(1):27-39.

35. Kapoor S, Kobayashi A, Takatsuji H. Silencing of the tapetum-specific zinc finger gene TAZ1 causes premature degeneration of tapetum and pollen abortion in petunia. Plant Cell. 2002;14(10):2353-67.

36. Sorensen AM, Kröber S, Unte US, Huijser P, Dekker K, Saedler H. The Arabidopsis aborted microspores ( AMS ) gene encodes a MYC class transcription factor. Plant J. 2003;33(2):413-23.

37. Higginson T, Li SF, Parish RW. AtMYB103 regulates tapetum and trichome development in Arabidopsis thaliana. Plant J. 2003;35(2):177-92.

38. Zhang W, Sun Y, Timofejeva L, Chen C, Grossniklaus U, Ma H. Regulation of Arabidopsis Tapetum development and function by dysfunctional tapetum1 (DYT1) encoding a putative bHLH transcription factor. Development. 2006; 133(16):3085-95.

39. Feng BM, Lu DH, Ma X, Peng YB, Sun YJ, Ning G, et al. Regulation of the Arabidopsis anther transcriptome by DYT1 for pollen development. Plant J. 2012;72(4):612-24.

40. Hsieh K, Huang AH. Tapetosomes in brassica tapetum accumulate endoplasmic reticulum-derived flavonoids and alkanes for delivery to the pollen surface. Plant Cell. 2007;19(2):582-96.

41. Choi H, Jin JY, Choi S, Hwang JU, Kim YY, Mi CS, et al. An ABCG/WBC-type $A B C$ transporter is essential for transport of sporopollenin precursors for exine formation in developing pollen. Plant J. 2011;65(2):181-93.

42. Choi H, Ohyama K, Kim YY, Jin JY, Lee SB, Yamaoka Y, et al. The role of ArabidopsisABCG9 and ABCG31 ATP binding cassette transporters in pollen fitness and the deposition of steryl glycosides on the pollen coat. Plant Cell. 2014;26(1):310-24.

43. Parish RW, Li SF. Death of a tapetum. A programme of developmental altruism. Plant Sci. 2010;178(2):73-89.

44. Solomon M, Belenghi B, Delledonne M, Menachem E, Levine A. The involvement of cysteine proteases and protease inhibitor genes in the regulation of programmed cell death in plants. Plant Cell. 1999;11(3):431-44

45. Zhang DD, Liu D, Lv XM, Wang Y, Xun ZL, Liu ZX, et al. The cysteine protease CEP1, a key executor involved in tapetal programmed cell death, regulates pollen development in Arabidopsis. Plant Cell. 2014;26(7):2939-61.

46. Martin C. Transcription factors and the manipulation of plant traits. Curr Opin Biotechnol. 1996;7(7):130-38.
47. Dubos C, Stracke R, Grotewold E, Weisshaar B, Martin C, Lepiniec L. MYB transcription factors in Arabidopsis. Trends Plant Sci. 2010:15(10):573-81.

48. Eckardt NA. Elucidating the function of synergid cells: a regulatory role for MYB98. Plant Cell. 2007;19(8):2320-21.

49. Kasahara RD, Portereiko MF, Sandaklie-Nikolova L, Rabiger DS, Drews GN. MYB98 is required for pollen tube guidance and synergid cell differentiation in Arabidopsis. Plant Cell. 2005;17(11):2981-92.

50. Liang Y, Tan ZM, Zhu L, Niu QK, Zhou JJ, Li M, et al. MYB97, MYB101 and MYB120 function as male factors that control pollen tube-synergid interaction in Arabidopsis thaliana fertilization. PLoS Genet. 2013;9(11): e1003933.

51. Messenguy F, Dubois E. Role of MADS box proteins and their cofactors in combinatorial control of gene expression and cell development. Gene. 2003;316:1-21.

52. Huang F, Xu GL, Chi YJ, Liu HC, Xue Q, Zhao TJ, et al. A soybean MADS-box protein modulates floral organ numbers, petal identity and sterility. BMC Plant Biol. 2014;14:89.

53. Levy YY, Mesnage S, Mylne JS, Gendall AR, Dean C. Multiple roles of Arabidopsis VRN1 in vernalization and flowering time control. Science. 2002; 297(5579):243-46.

54. Huang WC, Hu J, Yu CC, Huang Q, Wan L, Wang LL, et al. Two non-allelic nuclear genes restore fertility in a gametophytic pattern and enhance abiotic stress tolerance in the hybrid rice plant. Theor Appl Genet. 2012; 124(5):799-807.

55. Yasumoto $K$, Terachi T, Yamagishi $H$. A novel Rf gene controlling fertility restoration of Ogura male sterility by RNA processing of orf138 found in Japanese wild radish and its STS markers. Genome. 2009;52(6):495-504.

56. Jo YD, Kim YM, Park MN, Yoo JH, Park MK, Kim BD, et al. Development and evaluation of broadly applicable markers for restorer-of-fertility in pepper. Mol Breed. 2010;25(2):187-201.

57. Holzle A, Jonietz C, Torjek O, Altmann T, Binder S, Forner J. A restorer of fertility-like $P P R$ gene is required for $5^{\prime}$-end processing of the nad4 mRNA in mitochondria of Arabidopsis thaliana. Plant J. 2011;65(5):737-44.

58. Krueger F, Andrews SR. Bismark: a flexible aligner and methylation caller for bisulfite-Seq applications. Bioinformatics. 2011;27(11):1571-72.

59. Smallwood SA, Lee HJ, Angermueller C, Krueger F, Saadeh H, Peet J, et al. Single-cell genome-wide bisulfite sequencing for assessing epigenetic heterogeneity. Nat Methods. 2014;11(8):817-20.

60. Lister R, Mukamel EA, Nery JR, Urich M, Puddifoot CA, Johnson ND, et al. Global epigenomic reconfiguration during mammalian brain development. Science. 2013;341(6146):1237905

61. Wang Z, Li XF, Jiang Y, Shao QZ, Liu Q, Chen BY, Huang DS. swDMR: A sliding window approach to identify differentially methylated regions based on whole genome bisulfite sequencing. PloS One. 2015;10(7):e0132866.

62. Young MD, Wakefield MJ, Smyth GK, Oshlack A. Gene ontology analysis for RNA-seq: accounting for selection bias. Genome Biol. 2010;11(2):R14.

63. Mao XZ, Cai T, Olyarchuk JG, Wei LP. Automated genome annotation and pathway identification using the KEGG Orthology (KO) as a controlled vocabulary. Bioinformatics. 2005;21(19):3787-93.

64. Li YY, Tollefsbol TO. DNA methylation detection: bisulfite genomic sequencing analysis. Methods Mol Biol. 2011;791:11-21.

65. Trapnell C, Hendrickson DG, Sauvageau M, Goff L, Rinn JL. Differential analysis of gene regulation at transcript resolution with RNA-seq. Nat Biotechnol. 2013;31(1):46-53.

66. Benjamini BY, Yekutieli D. The control of the false discovery rate in multiple testing under dependency. Ann Stat. 2001;29:1165-88.

67. Benjamini BY, Hochberg Y. Controlling the false discovery rate: a practical and powerful approach to multiple testing. J R Stat Soc. 1995;57:289-300. 